\title{
The full-spectrum correlated-k method for longwave atmospheric radiative transfer using an effective Planck function
}

Article

Published Version

Hogan, R. J. (2010) The full-spectrum correlated-k method for longwave atmospheric radiative transfer using an effective Planck function. Journal of the Atmospheric Sciences, 67 (6). pp. 2086-2100. ISSN 1520-0469 doi:

https://doi.org/10.1175/2010JAS3202.1 Available at https://centaur.reading.ac.uk/16211/

It is advisable to refer to the publisher's version if you intend to cite from the work. See Guidance on citing.

To link to this article DOI: http://dx.doi.org/10.1175/2010JAS3202.1

Publisher: American Meteorological Society

All outputs in CentAUR are protected by Intellectual Property Rights law, including copyright law. Copyright and IPR is retained by the creators or other copyright holders. Terms and conditions for use of this material are defined in the End User Agreement.

www.reading.ac.uk/centaur 
Central Archive at the University of Reading

Reading's research outputs online 


\title{
The Full-Spectrum Correlated- $k$ Method for Longwave Atmospheric Radiative Transfer Using an Effective Planck Function
}

\author{
ROBIN J. HOGAN \\ Department of Meteorology, University of Reading, Reading, United Kingdom
}

(Manuscript received 21 May 2009, in final form 22 January 2010)

\begin{abstract}
The correlated- $k$-distribution (CKD) method is widely used in the radiative transfer schemes of atmospheric models; it involves dividing the spectrum into a number of bands and then reordering the gaseous absorption coefficients within each one. The fluxes and heating rates for each band may then be computed by discretizing the reordered spectrum into $O(10)$ quadrature points per major gas and performing a pseudomonochromatic radiation calculation for each point. In this paper it is first argued that for clear-sky longwave calculations, sufficient accuracy for most applications can be achieved without the need for bands: reordering may be performed on the entire longwave spectrum. The resulting full-spectrum correlated- $k$ (FSCK) method requires significantly fewer pseudomonochromatic calculations than standard CKD to achieve a given accuracy. The concept is first demonstrated by comparing with line-by-line calculations for an atmosphere containing only water vapor, in which it is shown that the accuracy of heating rate calculations improves approximately in proportion to the square of the number of quadrature points. For more than around 20 points, the root-mean-square error flattens out at around $0.015 \mathrm{~K} \mathrm{day}^{-1}$ due to the imperfect rank correlation of absorption spectra at different pressures in the profile. The spectral overlap of $m$ different gases is treated by considering an $m$-dimensional hypercube where each axis corresponds to the reordered spectrum of one of the gases. This hypercube is then divided up into a number of volumes, each approximated by a single quadrature point, such that the total number of quadrature points is slightly fewer than the sum of the number that would be required to treat each of the gases separately. The gaseous absorptions for each quadrature point are optimized such that they minimize a cost function expressing the deviation of the heating rates and fluxes calculated by the FSCK method from line-by-line calculations for a number of training profiles. This approach is validated for atmospheres containing water vapor, carbon dioxide, and ozone, in which it is found that in the troposphere and most of the stratosphere, heating rate errors of less than $0.2 \mathrm{~K} \mathrm{day}^{-1}$ can be achieved using a total of 23 quadrature points, decreasing to less than $0.1 \mathrm{~K} \mathrm{day}^{-1}$ for 32 quadrature points. It would be relatively straightforward to extend the method to include other gases.
\end{abstract}

\section{Introduction}

A prerequisite for reliable modeling of the climate system is the ability to calculate accurate heating rate profiles and surface fluxes, including the effects of small changes to the concentrations of greenhouse gases. However, the longwave gaseous absorption spectrum exhibits significant structure over spectral intervals a millionth of the range over which terrestrial emission is significant, implying that $O\left(10^{6}\right)$ monochromatic calculations are required. Ambartzumian (1936) was the first to propose that, for vertically homogeneous atmospheres, the gaseous mass

Corresponding author address: Robin J. Hogan, Department of Meteorology, Earley Gate, P.O. Box 243, Reading RG6 6BB, United Kingdom.

E-mail: r.j.hogan@reading.ac.uk. absorption coefficients $k$ can be "sorted" into a monotonic function that is much more conducive to efficient numerical integration. This was extended to vertically inhomogeneous atmospheres by Lacis et al. (1979), and the resulting "correlated- $k$ distribution" (CKD) method now forms the basis of most radiative transfer schemes in general circulation models (GCMs). It takes advantage of the fact that in the terrestrial atmosphere the ordering of the spectrum is highly correlated in the vertical, despite large changes in absorber abundance and the effect of changes to the spectral lines through pressure and Doppler broadening.

Even though CKD methods are so much more efficient than line-by-line calculations, they can still constitute a significant fraction of the computational cost of a GCM. High spectral accuracy is therefore usually achieved at the expense of poor temporal resolution, with 
the radiation scheme often called only every $3 \mathrm{~h}$, which can lead to errors in the diurnal cycle (Yang and Slingo 2001) and can change the climate sensitivity of the model (Morcrette 2000). The computational time spent resolving the spectrum is also not matched by the time spent resolving the spatial structure of clouds within a grid box, leading to substantial radiative biases (Cahalan et al. 1994; Shonk and Hogan 2008). Moreover, while it is necessary to represent trace gases individually for climate forecasts and reanalysis projects, the accuracy of day-to-day numerical weather forecasts is largely insensitive to errors in their representation; Curry et al. (2006) showed that assuming trace gases to be vertically well mixed led to a temperature error of less than $0.2 \mathrm{~K}$ below $30 \mathrm{~km}$, rising to $1 \mathrm{~K}$ above $50 \mathrm{~km}$. It is therefore highly desirable to explore ways to treat gaseous absorption more efficiently.

Most current longwave CKD models divide the spectrum into bands and then reorder the spectrum within each band. The number of bands can vary considerably. The model presented by Lacis and Oinas (1991) used narrow $10 \mathrm{~cm}^{-1}$ spectral intervals and 36 quadrature points within each, resulting in a total of around $10^{4}$ quadrature points. The Rapid Radiative Transfer Model (RRTM) of Mlawer et al. (1997) employs 16 bands in the longwave with a total of 256 quadrature points [reduced to 140 in its implementation in the model of the European Centre for Medium-Range Weather Forecasts, Morcrette et al. (2008)], while Fu and Liou (1992) demonstrated that sufficient accuracy could be achieved with 67 points in 12 bands. Fomin (2004) reported a CKD model employing only 23 points in 4 bands, although the heating rate errors up to $0.9 \mathrm{~K} \mathrm{day}^{-1}$ in the stratosphere are probably too large for this model to be considered for operational use.

So what determines the number of bands into which it is necessary to divide the spectrum? The following considerations have been cited previously:

1) The need for the band to be narrow enough that the Planck function does not vary significantly across it (Fu and Liou 1992).

2) The need to minimize the number of active gases within each band owing to the computational expense of treating overlapping gases (Mlawer et al. 1997). Moreover, some techniques for treating gaseous overlap, such as fast exponential sum fitting of transmissivities (FESFT) (Ritter and Geleyn 1992; Edwards 1996), assume that the overlap is random, which is not valid over very wide spectral intervals.

3) The need to resolve the spectral variation of cloud and aerosol absorption and scattering, which varies much more slowly than gaseous absorption (Ritter and Geleyn 1992).
A recent proposal for the rapid computation of radiative transfer within combusting gases is the full-spectrum correlated-k (FSCK) method in which the entire spectrum is reordered as a single band (Modest and Zhang 2002) and the continuous distribution of gaseous absorption is discretized into a smaller total number of quadrature points than is required with bands. They tackled the first consideration above by weighting each of the quadrature points by the integral of the Planck function at some reference temperature of each part of the spectrum contributing to that point. Other temperatures were treated using lookup tables to relate the absorption between different temperatures. FSCK has been applied to the shortwave by Pawlak et al. (2004). With the absence of internal emission, Pawlak et al. were able simply to weight each quadrature point by the intensity of solar radiation contributing to it (although this is also done in many CKD models using narrower bands; e.g., Fu and Liou 1992). For representing clouds, they found it necessary to divide the shortwave region into two bands on either side of a wavelength of $0.68 \mu \mathrm{m}$; at shorter wavelengths than this clouds have virtually zero absorption, while at longer wavelengths absorption becomes significant.

In the longwave, a method essentially the same as FSCK has been applied in the Goddard Institute for Space Studies (GISS) GCM (A. Lacis 2009, personal communication) for many years. The original version was the one used by Hansen et al. (1983) and Lacis and Oinas (1991) and employed 25 quadrature points. The current GISS radiation scheme uses a total of 33 quadrature points (13 for $\mathrm{H}_{2} \mathrm{O}, 12$ for $\mathrm{CO}_{2}$, and 8 for $\mathrm{O}_{3}$ ), and its first reported use was by Oinas et al. (2001). The cloud optical properties are merged with the gaseous correlated- $k$ distributions. However, none of these papers discuss in any detail how optimal selection of these bands is performed or how the tricky problem of the spectral overlap of gases is tackled across the full spectrum. Furthermore, it is the author's experience that many radiation experts are unaware that it is possible to apply the CKD method to the full spectrum.

The purpose of this paper is to demonstrate the application of the FSCK method to atmospheric longwave radiative transfer, focusing particularly on how to optimize the selection of quadrature points and the treatment of the spectral overlap of multiple gases. To treat consideration 1 above, we take a simpler approach than in Modest and Zhang (2002) and replace occurrences of the Planck function in the equations of radiative transfer by an effective Planck function, which represents the integral of the Planck function over those parts of the spectrum contributing to a particular quadrature point (which may be from opposite ends of the spectrum). In 
section 2 we outline mathematically the origin of the FSCK approach and demonstrate that the use of an effective Planck function is exact, subject to the ordering of absorption spectra being perfectly correlated along a path. In section 3, the numerical convergence of the method is tested by comparison with line-by-line calculations for an atmosphere containing only a single gas $\left(\mathrm{H}_{2} \mathrm{O}, \mathrm{CO}_{2}\right.$, or $\left.\mathrm{O}_{3}\right)$, quantifying the effect of imperfect correlation in the vertical due to broadening of the spectral lines. In section 4 , a method to treat the spectral overlap of many gases across the spectrum is presented, which is then tested on atmospheres containing $\mathrm{H}_{2} \mathrm{O}$, $\mathrm{CO}_{2}$, and $\mathrm{O}_{3}$ in section 5. It should be stressed that the purpose of this paper is not to present a finalized radiative transfer model, ready to be implemented in climate models, but rather to demonstrate the promise of the technique; in section 6 , we discuss the remaining work to be done before such a model could be written.

\section{Theoretical background}

To facilitate the discussion of the correlated- $k$ method and its full-spectrum counterpart, it is convenient to simplify the equations of longwave radiative transfer. If the atmosphere is discretized vertically into $n_{z}$ layers, then the heating rate of layer $i$ may be written as

$$
\dot{T}_{i}=\int_{\eta_{\min }}^{\eta_{\max }} \sum_{j=0}^{n_{z}} \mathbf{w}_{j i}(\eta) B_{j}(\eta) d \eta,
$$

where $B_{j}(\eta)$ is the Planck function $\left[\mathrm{W} \mathrm{m}{ }^{-2}\left(\mathrm{~cm}^{-1}\right)^{-1}\right.$ ] at wavenumber $\eta$ for the temperature of layer $j$ : $\mathbf{W}_{j i}(\eta)$ is a weighting matrix $\left(\mathrm{K} \mathrm{m}^{2} \mathrm{~J}^{-1}\right)$ that accounts for all aspects of the transmission between layer $j$ and layer $i$ at wavenumber $\eta$, integrated over angle, but multiplied by additional factors to convert from irradiance to heating rate. In the case that $j=0$, the factor $\mathbf{W}_{0 i}(\eta)$ should be thought of as the transmission from the surface [with blackbody emission $B_{0}(\eta)$ ] to layer $i$, whereas in the case of $j=i$, the factor $\mathbf{W}_{i i}(\eta)$ represents the ability of the layer to lose thermal energy by emission.

In the appendix it is shown how $\mathbf{W}_{j i}$ may be calculated explicitly, including how to include the effects of scattering from other layers in the transit of radiation from layer $j$ to layer $i$. Thus, the $\sum_{j=0}^{n_{z}} \mathbf{W}_{j i}(\eta) B_{j}(\eta)$ term simply represents a monochromatic radiative transfer calculation using the two-stream or other appropriate method. The key point to note from (1) is that, even when scattering is present, the longwave heating rate of a layer can still be considered as the linear sum of the Planck function of all layers of the atmosphere, weighted appropriately. Equation (1) is similar to the formulation of longwave radiative transfer in terms of net exchange matrices (Green 1967; Eymet et al. 2004). However, it is more general in the sense that we could write equations for the fluxes at any point in the atmosphere in exactly the same form: the linear sum of weighted contributions from the Planck function at every point in the atmosphere.

The integral in (1) is performed over the wavenumber range of the entire longwave spectrum from $\eta_{\min } \sim$ $100 \mathrm{~cm}^{-1}$ to $\eta_{\max } \sim 2500 \mathrm{~cm}^{-1}$. Throughout this section we use summations to indicate when fewer than around 100 elements are being summed but employ integrals when more than around $10^{4}$ quadrature points would be required for a numerical integration.

\section{a. The correlated-k method}

The rapid spectral variation of gaseous absorption, and hence the rapid variation of $\mathbf{W}_{j i}$ with $\eta$, means that of $O\left(10^{6}\right)$ monochromatic radiative transfer calculations would be required to evaluate the integral in (1). Such "line-by-line" calculations are essential to provide accurate benchmarks, but are too expensive for use in a GCM. The correlated-k distribution method is a widely used approximation to make the spectral integration more efficient. The spectrum is split up into $n_{\bar{\eta}}$ bands within which the Planck function may be assumed constant with wavenumber, although many CKD models do account for the variation of the Planck function within a band. Within each band, the spectrum is reordered in terms of the mass absorption coefficient $k$ such that a rapidly varying function of $\eta$ is replaced with a monotonically increasing function of the "normalized rank" $g$, which varies from 0 for the least absorbing part of the band to 1 for the most absorbing part. Therefore, within each band a summation is performed in $g$ space, and (1) becomes

$$
\dot{T}_{i}=\sum_{m=1}^{n_{\bar{\eta}}} \sum_{l=1}^{n_{g}} \sum_{j=0}^{n_{z}} \mathbf{W}_{j i}\left(g_{l m}\right) B_{j}(\bar{\eta}) \Delta g_{l m} \Delta \bar{\eta}_{m},
$$

where $n_{g}$ separate pseudomonochromatic calculations are performed in each band, each weighted by their width in $g$ space, $\Delta g_{l m}$. Since the absorption varies slowly with $g$, far fewer points need to be used in the integration (typically between 2 and 16).

\section{b. The full-spectrum correlated-k (FSCK) method}

We now consider the basis behind the full-spectrum correlated- $k$ method. Rather than splitting the spectrum up into bands, we order the entire longwave spectrum by absorption coefficient such that the value of $g$ now indicates the rank with respect to all other values in the spectrum. Thus $g(\eta)$ represents a nondifferentiable function relating wavenumber to normalized rank, and 
$\eta(g)$ is its inverse. The first step is a change of variables in the integration from $\eta$ to $g$ such that (1) becomes

$$
\dot{T}_{i}=\int_{0}^{1} \sum_{j=0}^{n_{z}} \mathbf{W}_{j i}(g) B_{j}[\eta(g)] d g .
$$

The main difference is that in (1) $\mathbf{W}_{j i}$ is a rapidly varying function of the variable of integration $\eta$, while $B_{j}$ is a smooth function of $\eta$. Conversely, in (3) $\mathbf{W}_{j i}$ is a smoothly varying function of the variable of integration $g$ whereas $B_{j}$ varies rapidly with $g$. The integral over $g$ presents a small conceptual problem since one could argue that there are many wavenumbers corresponding to a given $g$. However, if the integral is thought of as a discrete summation of arbitrarily fine resolution, then each $g$ in the summation corresponds to a unique wavenumber and the conceptual problem is overcome. We retain the integral sign in (3) to stress that a very high resolution summation would be required to evaluate it.

We now discretize the $g$ distribution into $n_{g}$ intervals (where $n_{g}$ is again $O(10)$ such that interval $l$ is bounded by the values $g_{l-1 / 2}$ and $g_{l+1 / 2}$ and within the interval the weighting factor $\mathbf{W}_{j i}\left(g_{l}\right)$ is constant. Thus (3) becomes

$$
\dot{T}_{i}=\sum_{l=1}^{n_{g}} \int_{g_{l-1 / 2}}^{g_{l+1 / 2}} \sum_{j=0}^{n_{z}} \mathbf{W}_{j i}\left(g_{l}\right) B_{j}[\eta(g)] d g .
$$

We are still left with the problem that a particular narrow range of $g$ now includes disparate parts of the spectrum, and therefore it is not valid to choose a representative wavenumber $\bar{\eta}$ for calculating the Planck function, as was the case within a band in the formulation of (2). The approach taken by Modest and Zhang (2002) was indeed to use an integrated Planck function for the entire wavenumber range $\eta_{\min }-\eta_{\max }$ in the pseudomonochromatic radiative transfer calculations but to weight the transmittances $\left(\mathbf{W}_{j i}\right.$ in our terminology) by the Planck function at the parts of the spectrum that contribute to the range of $g$ under consideration at an arbitrary reference temperature. Lookup tables were then required to try to account for variations of the weighting at different temperatures along the path. However, a simpler approach is possible.

\section{c. FSCK with an effective Planck function}

In (4) it can be seen that $\mathbf{W}_{i i}\left(g_{l}\right)$ is constant over the range of $g$ represented in the integration, and hence the integral can be applied directly to the Planck function as follows:

$$
\begin{aligned}
\dot{T}_{i} & =\sum_{l=1}^{n_{g}} \sum_{j=0}^{n_{z}} \mathbf{W}_{i j}\left(g_{l}\right) \int_{g_{l-1 / 2}}^{g_{l+1 / 2}} B_{j}[\eta(g)] d g \\
& =\sum_{l=1}^{n_{g}} \sum_{j=0}^{n_{z}} \mathbf{w}_{i j}\left(g_{l}\right) B_{j}^{\prime}\left(g_{l}\right) .
\end{aligned}
$$

In (5), we have introduced an effective Planck function $B_{j}^{\prime}\left(g_{l}\right)$, which is simply the integral of the Planck function over the wavenumbers that contribute to absorption in a particular range of $g$. Comparing (5) to (1), we see that integration over $g$ is identical to integration over $\eta$ except that we have only $n_{g}$ pseudomonochromatic radiative transfer calculations to perform (where $n_{g}$ is far smaller than the $\sim 10^{6}$ required to resolve the spectrum): we must use the effective Planck function in place of the conventional Planck function wherever it appears in the radiative transfer calculation. The effective Planck function is straightforward to implement as a lookup table versus temperature for each of the $n_{g}$ quadrature points.

To summarize, what has been shown in this section is that different parts of the spectrum with similar absorption properties may be treated together in a single radiative transfer calculation, provided that occurrences of the Planck function in the calculation are replaced by the integral of the Planck function over those parts of the spectrum being considered. This approach is already taken over limited parts of the spectrum in some CKD models (e.g., RRTM), but it has been shown here to be equally valid over the entire longwave spectrum.

\section{Results for a single absorbing gas}

We first test the validity of (5) using the standard midlatitude summer (MLS) atmosphere of McClatchey et al. (1972) but containing only a single absorbing gas. Absorption spectra of water vapor, carbon dioxide, and ozone have been calculated at a resolution of $0.0025 \mathrm{~cm}^{-1}$ using the High-Resolution Transmission Molecular Absorption (HITRAN) database (Rothman et al. 2004) coupled to the line-by-line model of Mitsel et al. (1995) assuming Voigt line shapes. The MT-CKD water vapor continuum of Clough et al. (2005) is included. Spectra have been calculated at reference pressures of $0.01,0.1,1,10,30$, 100,500 , and $1013 \mathrm{hPa}$ for temperature and humidity conditions similar to the MLS atmosphere.

A benchmark radiative transfer calculation with a spectral resolution of $0.0025 \mathrm{~cm}^{-1}$ is first performed for the case in which water vapor is the only absorbing gas. The atmosphere is represented with a resolution of $1 \mathrm{~km}$ below $25 \mathrm{~km}$ and $5 \mathrm{~km}$ above, up to the highest model level at $100 \mathrm{~km}$. Mass absorption coefficients are interpolated logarithmically in logarithmic pressure space from the reference spectra. The two-stream approximation in the absence of scattering is employed, in which for each wavenumber interval a single upwelling and a single downwelling path is followed through the atmosphere at zenith angles of $\pm 52^{\circ}$ (e.g., Fu et al. 1997), and emission and absorption are treated within each layer, 


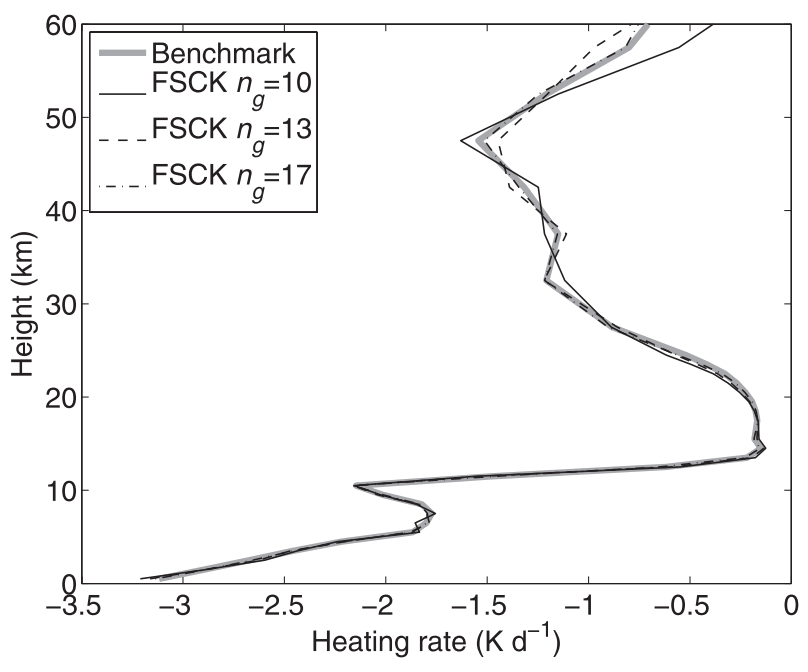

FIG. 1. Heating rate in the midlatitude summer atmosphere in which water vapor is the only absorbing gas. Results are shown for a benchmark calculation using a spectral resolution of $0.0025 \mathrm{~cm}^{-1}$ (thick gray line) and using the full-spectrum correlated- $k$ method (FSCK) with three different numbers of quadrature points $n_{g}$ (thin black lines). The $n_{g}$ values of 10,13 , and 17 were generated by assigning error tolerances $\sigma_{\max }$ for each $g$ interval of $0.05,0.03$, and $0.02 \mathrm{~K} \mathrm{day}^{-1}$, respectively.

assuming the Planck function to vary linearly with optical depth. The surface emissivity is taken to be unity. Although the accuracy of the two-stream approximation is not commensurate with the spectral accuracy of a lineby-line calculation, the FSCK parameterization that will be developed would invariably be used with a two-stream radiation scheme and, therefore, should be compared against a benchmark that uses the same approximation. The benchmark heating rate profile is shown by the thick gray line in Fig. 1 and covers the longwave spectral range $100-2500 \mathrm{~cm}^{-1}(4-100 \mu \mathrm{m})$.

We next describe the derivation of the parameters of the FSCK model. The first task is to reorder the entire water vapor spectrum by $k$. The best way to do this is not immediately obvious since the optimum sorting is a function of pressure (and to a lesser extent temperature), and it is found that sorting just by $k$ at one pressure can lead to significant heating rate errors at other pressures. For example, sorting by the zenith optical depth weights the lower troposphere too much and results in large errors in the stratosphere. This problem is likely to be more severe for FSCK than CKD since a wider range of absorptions are being sorted simultaneously. To solve this problem, we perform a high-spectral-resolution radiative transfer calculation for an atmosphere with gas concentration from the MLS atmosphere but with a linearly decreasing temperature from the surface to an altitude of $100 \mathrm{~km}$. The spectrum is then sorted by the height at which the peak cooling rate occurs. This has the advantage that it provides optimal sorting at the pressure where each part of the spectrum is most important. Using a linearly decreasing temperature ensures that this method is not affected by the location of changes in the sign of the temperature gradient that are present in the real atmosphere. The method fails for optically thin parts of the spectrum, so, when the zenith optical depth $\delta$ is less than unity, the sorting is simply performed by $\delta$ instead. Thus we arrive at a normalized rank $g(\eta)$ for the entire spectrum. The black line in Fig. 2a displays this function for water vapor (the other two gases will be discussed shortly).

The second task in deriving the FSCK model parameters is to choose optimal intervals in $g$ space such that each interval is associated with approximately the same heating rate error. This is achieved by choosing the required error tolerance $\sigma_{\max }$ (e.g., $0.05 \mathrm{~K} \mathrm{day}^{-1}$ ) and then proceeding up through $g$ space. To calculate the parameters for interval 1 , we set the lower boundary to $g_{1 / 2}=0$ and test a particular value for the upper boundary $g_{3 / 2}$. Those parts of the high-resolution water vapor spectrum that lie in the range $g_{1 / 2}<g \leq g_{3 / 2}$ are selected, and at each height (plus the surface) the effective Planck function is calculated following (5). A first guess is then made of the best $k$ to use at each of the reference pressures listed above, by calculating the Planck-functionweighted mean of $\ln k$ for the same range of $g$. These are then used to calculate a profile of absorption coefficient for the MLS atmosphere. Radiative transfer calculations are then performed using the same twostream approximation as the benchmark calculations, for not only this first guess of the absorption profile but also for the absorption profiles scaled by several hundred different factors between 0.1 and 10 . The resulting heating-rate profiles $\dot{T}^{\mathrm{FSCK}}$ and fluxes are then compared to the benchmark heating rate profile $\dot{T}^{\text {bench }}$ and fluxes for the $g$ interval under consideration, and a root-mean-square error $\sigma$ is calculated for each scaling factor.

At this point it is worth discussing how the error is weighted since this can have significant consequences for the resulting model parameters. If each layer of the atmosphere is weighted by the pressure difference across it (i.e., its mass), then the troposphere will be weighted around four times more than the rest of the atmosphere, resulting in large errors being tolerated in the stratosphere. If each layer is weighted by its physical depth, then the stratosphere will be weighted around four times more than the troposphere, resulting in large tropospheric errors. Therefore, we weight each layer by the difference in the square root of the pressure $p$ at the boundaries of the layer, which results in the troposphere 

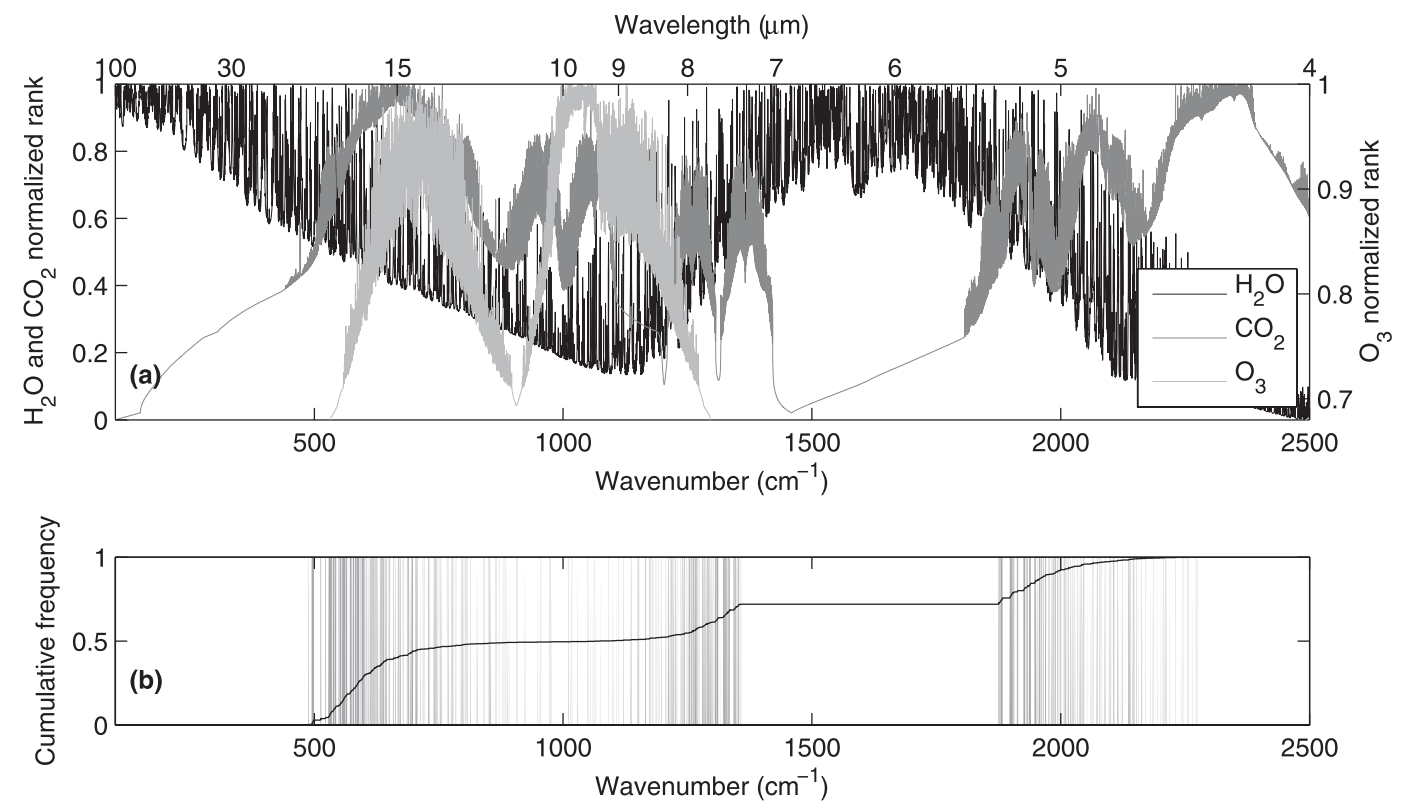

FIG. 2. (a) The normalized ranks of the spectra of water vapor, carbon dioxide, and ozone $\left(g^{\mathrm{H}_{2} \mathrm{O}}, g^{\mathrm{CO}_{2}}\right.$ and $\left.g^{\mathrm{O}_{3}}\right)$. Note that the scale for ozone is shown on the right, and the absorption coefficient of ozone outside the range $500-1295 \mathrm{~cm}^{-1}$ has been set to zero because of the dominance of the other two gases at other wavenumbers; hence, $\mathrm{g}_{3}$ is only shown above 0.68 . (b) The gray vertical lines indicate the parts of the water vapor spectrum for which the normalized rank lies in the fourth interval in Table 1 (i.e., $0.461<g^{\mathrm{H}_{2} \mathrm{O}} \leq 0.534$ ). The black line shows the corresponding cumulative frequency.

and stratosphere being weighted approximately equally. An additional term is added to penalize errors in the net fluxes at the top of the atmosphere (TOA), $F_{\mathrm{TOA}}$, and at the surface, $F_{\text {surf }}$. Thus, the error variance (which can be thought of as a cost function) is defined as

$$
\begin{aligned}
\sigma^{2}= & \frac{\sum_{i=1}^{n_{z}^{\prime}}\left(\dot{T}_{i}^{\mathrm{FSCK}}-\dot{T}_{i}^{\mathrm{bench}}\right)^{2}\left(p_{i-1 / 2}^{0.5}-p_{i+1 / 2}^{0.5}\right)}{p_{1 / 2}^{0.5}-p_{n_{z}^{\prime}+1 / 2}^{0.5}} \\
& +f\left[\left(F_{\mathrm{TOA}}^{\mathrm{FSCK}}-F_{\mathrm{TOA}}^{\mathrm{bench}}\right)^{2}+\left(F_{\text {surf }}^{\mathrm{FSCK}}-F_{\text {surf }}^{\mathrm{bench}}\right)^{2}\right],
\end{aligned}
$$

where $n_{z}^{\prime}$ is the index of the highest level that contributes to the error. In this study we use a threshold pressure of $0.1 \mathrm{hPa}(67 \mathrm{~km}$ in the MLS atmosphere), which is the typical pressure of the highest model level in a GCM (e.g., Météo-France has its highest level centered at $0.1 \mathrm{hPa}$, the ECMWF at $0.01 \mathrm{hPa}$, and the UK Met Office at $40 \mathrm{~km}$ ). Furthermore, at pressures lower than $0.1 \mathrm{hPa}$ the assumption of local thermodynamic equilibrium starts to become questionable. Of course, if mesospheric heating rates were of interest then $n_{z}^{\prime}$ could be increased. The factor $f$ weights the importance of the fluxes with respect to the heating rates. It is found that a value of $f=0.02\left(\mathrm{~K} \text { day }^{-1} \mathrm{~W}^{-1} \mathrm{~m}^{2}\right)^{2}$ provides the best balance of ensuring the flux errors are close to zero at the boundaries without compromising the need to match the heating rate profile.

Thus we acquire an error for each value of scaling factor between 0.1 and 10 . The optimum absorption profile is then simply the one with the lowest error, which in practice almost always lies between 0.5 and 2: hence, the arbitrary bounds chosen for the range to search do not have an impact on the result. This error is then compared to the tolerance: if $\sigma>\sigma_{\max }$, then a lower value of $g_{3 / 2}$ is tried and the process is repeated, while, if $\sigma<0.8 \sigma_{\max }$, then a larger value of $g_{3 / 2}$ is tried. If $0.8 \sigma_{\max } \leq \sigma \leq \sigma_{\max }$, then $g_{3 / 2}$ is accepted, and the optimum values of $k$ at the set of reference pressures are stored. The process is repeated to find the next $g$ interval, and so on until the $g$ space is fully partitioned. Figure $2 b$ illustrates how the contributions to a particular $g$ interval are often from a wide range of locations in the spectrum.

The resulting longwave heating rate profiles for three different tolerances are shown by the thin black lines in Fig. 1. There appears to be rapid convergence with increasing numbers of intervals $n_{g}$. To quantify this more rigorously, the solid black line in Fig. 3 shows the rootmean-square error [calculated using Eq. (6) but this time on the total heating rate rather than for just one range of $g$, and neglecting the second term on the right-hand side for fluxes] versus $n_{g}$. Between $n_{g}=2$ and $n_{g}=20$, approximately second-order convergence is achieved; 


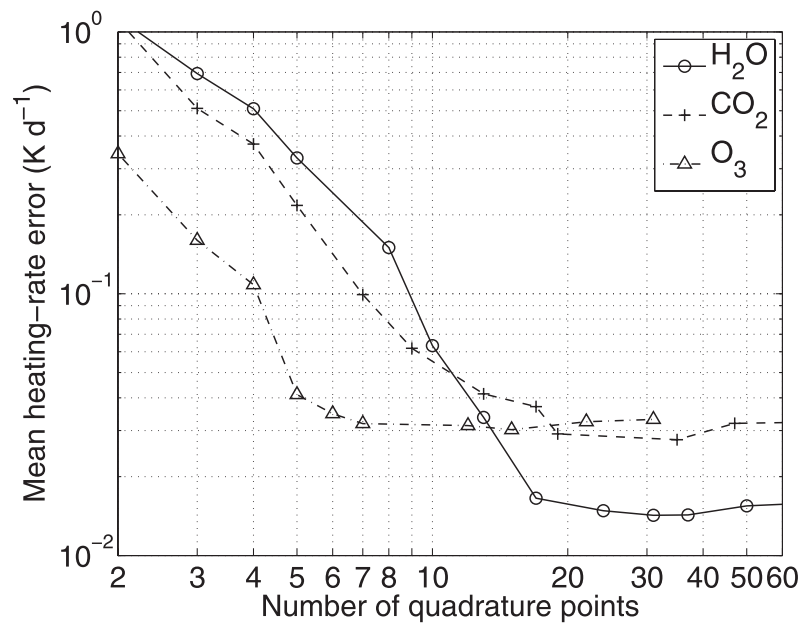

FIG. 3. Rms heating-rate error [weighted by the square root of pressure; i.e., the first term on the rhs of (6) for the FSCK method applied to the midlatitude summer standard atmosphere] vs the number of quadrature points used in the integration in $g$ space. The error is shown for an atmosphere containing $\mathrm{H}_{2} \mathrm{O}$ only, $\mathrm{CO}_{2}$ only, and $\mathrm{O}_{3}$ only (where for $\mathrm{O}_{3}$ only the absorption spectrum between wavenumbers of 500 and $1295 \mathrm{~cm}^{-1}$ is considered).

that is, the error is approximately proportional to $n_{g}^{-2}$. For $n_{g}>20$, the error flattens out at around $0.015 \mathrm{~K} \mathrm{day}^{-1}$. This is believed to be due to imperfect rank correlation of the $k$ values at one pressure with those at another.

The procedure is now repeated but with the only absorbing gas being carbon dioxide or ozone. Ozone absorption outside the wavenumber range $500-1295 \mathrm{~cm}^{-1}$ is negligible compared to absorption by water vapor and carbon dioxide; therefore, its absorption here is set to zero so that $g$ intervals are selected only in the important region. Then the ozone absorption in regions with $g^{\mathrm{O}_{3}}<0.9$ is set to zero for the same reason. Note that, when mixtures of different gases are tested, they are compared against high-spectral-resolution calculations in which the full ozone spectrum has been included. A well-mixed carbon dioxide profile is assumed with a concentration of $350 \mathrm{ppmv}$, while the ozone profile is taken from the MLS standard atmosphere. The results are shown in Fig. 3, where it can be seen that these two gases achieve a comparable order of convergence to water vapor, but flattening out at a larger error of around $0.03 \mathrm{~K} \mathrm{day}^{-1}$. This is believed to be due to the fact that the peak heating rates of carbon dioxide and ozone occur in the stratosphere and here they span a wider range of pressures than for water vapor. Thus, the error due to imperfect rank correlations at different pressures is larger. Ozone requires a smaller number of quadrature points than the other two gases to achieve the same accuracy simply because it is important over a much narrower range of the spectrum and over a smaller
TABLE 1. Optimum boundaries in $g$ space to achieve an error of less than $\sigma_{\max }=0.03 \mathrm{~K} \mathrm{day}^{-1}$ in each interval. The resulting 32-point model is discussed in the text.

\begin{tabular}{clll}
\hline$g$ boundary & \multicolumn{1}{c}{$\mathrm{H}_{2} \mathrm{O}$} & \multicolumn{1}{c}{$\mathrm{CO}_{2}$} & \multicolumn{1}{c}{$\mathrm{O}_{3}$} \\
\hline 0.5 & 0 & 0 & 0 \\
1.5 & 0.300 & 0.784 & 0.905 \\
2.5 & 0.405 & 0.871 & 0.982 \\
3.5 & 0.461 & 0.888 & 0.995 \\
4.5 & 0.534 & 0.913 & 0.9986 \\
5.5 & 0.622 & 0.936 & 0.99978 \\
6.5 & 0.721 & 0.964 & 1 \\
7.5 & 0.844 & 0.986 & \\
8.5 & 0.925 & 0.9931 & \\
9.5 & 0.974 & 0.9955 & \\
10.5 & 0.9939 & 0.9966 & \\
11.5 & 0.9983 & 0.9976 & \\
12.5 & 0.99943 & 0.9984 & \\
13.5 & 1 & 0.9989 & \\
14.5 & & 0.99947 & \\
15.5 & & 1 & \\
\hline
\end{tabular}

height range. Table 1 lists the boundaries of the $g$ intervals for each of the three gases in the case when the tolerance is set as $\sigma_{\max }=0.03 \mathrm{~K} \mathrm{day}^{-1}$.

\section{Results for more than one absorbing gas}

Figure 3 confirms that the FSCK method with an effective Planck function works well in the case of a single absorbing gas, but in the real atmosphere we must contend with the problem of spectrally overlapping gases. The issue is easiest to visualize by plotting the normalized rank of one gas versus that of another, as shown for water vapor and carbon dioxide in Fig. 4. The "brute force" approach would be to use the intervals of $g$ obtained for each gas in the previous spectrum, divide the space up into $n_{g}^{\mathrm{H}_{2} \mathrm{O}} \times n_{g}^{\mathrm{CO}_{2}}$ rectangles, and perform a pseudomonochromatic radiative transfer calculation for each. Clearly with any more than two gases, the number of pseudomonochromatic calculations becomes many more than would be required in an ordinary CKD calculation and the potential efficiency gains of FSCK would be lost.

Several techniques for reducing the number of pseudomonochromatic calculations have been proposed for overlapping gases. For example, FESFT (Ritter and Geleyn 1992; Edwards 1996) assumes that the spectra of the various gases are randomly overlapped, enabling each gas to be treated separately (akin to the one-gas calculations in the previous section), followed by the transmissivities of each gas being multiplied together. The number of pseudomonochromatic calculations required is then one plus the sum of the number required for each individual gas. However, this method is very inaccurate in an FSCK context because over the full spectrum the 


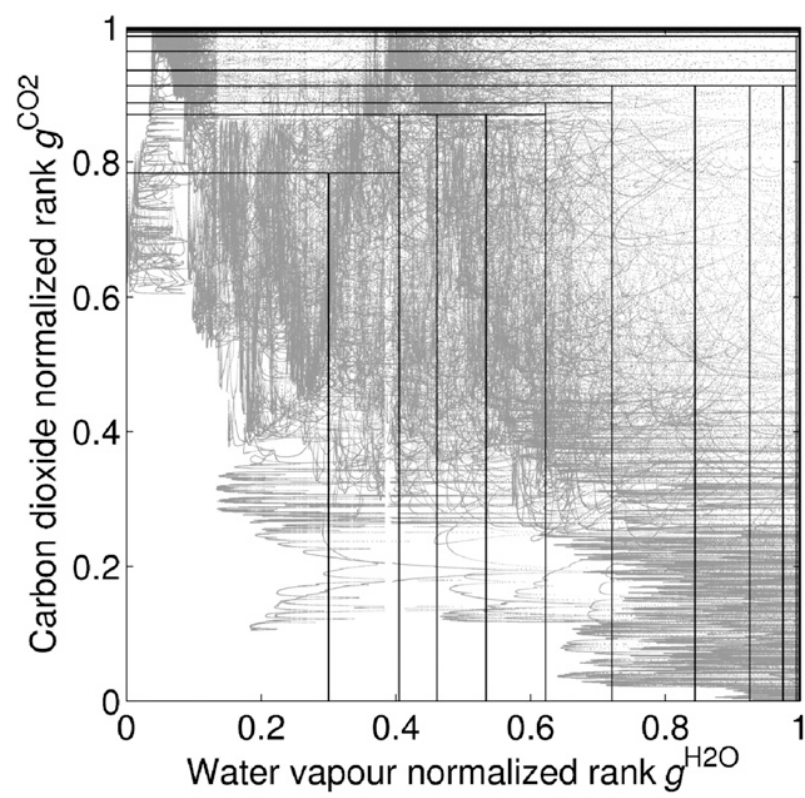

FIG. 4. Scatterplot of the entire longwave spectrum (100$2500 \mathrm{~cm}^{-1}$ ) where each point corresponds to a $0.0025 \mathrm{~cm}^{-1}$ interval, plotted as the normalized rank of the carbon dioxide absorption spectrum at that point $g^{\mathrm{CO}_{2}}$ vs the normalized rank of the water vapor spectrum $g^{\mathrm{H}_{2} \mathrm{O}}$. The rectangles show the regions of the spectrum that are treated together in the 32-point model discussed in the text.

spectral overlap is not perfectly random, and also because of the significant variation of the Planck function.

We therefore take a different approach. Examination of Fig. 4 reveals that the regions of highest water vapor absorption correspond to low carbon dioxide absorption; in particular, the bottom right of the figure is a combination of the vibration-rotation water vapor band centered at $6.7 \mu \mathrm{m}$ and the pure rotation band at wavelengths longer than around $20 \mu \mathrm{m}$. Similarly, regions of highest carbon dioxide absorption correspond to low or medium water vapor absorption; in particular, the concentration of points at the top left and top center of the figure correspond to the carbon dioxide bands at 4.3 and $15 \mu \mathrm{m}$, respectively. In these regions there is no need to resolve the variation in absorption of the weaker of the two absorbers since the fluxes and heating rates will be dominated by the stronger absorber.

Our procedure to partition $g$ space is as follows, considering first the case of two gases. The intention is generally to select regions in order of the height at which they are most important for the heating rate. The first region is defined to contain normalized ranks for the two gases $\left(g^{\mathrm{H}_{2} \mathrm{O}}\right.$ and $\left.g^{\mathrm{CO}_{2}}\right)$ such that $0<g^{\mathrm{H}_{2} \mathrm{O}} \leq g_{3 / 2}^{\mathrm{H}_{2} \mathrm{O}}$ and $0<$ $g^{\mathrm{CO}_{2}} \leq g_{3 / 2}^{\mathrm{CO}_{2}}$. This is shown as the rectangle in the lower left of Fig. 4 (note that the rectangle boundaries in this figure correspond to a tolerance of $\sigma_{\max }=0.03 \mathrm{~K} \mathrm{day}^{-1}$, resulting in $n_{g}^{\mathrm{H}_{2} \mathrm{O}}=13$ and $n_{g}^{\mathrm{CO}_{2}}=15$ ). To define the boundaries of the second region, the water vapor rank is advanced such that the second region lies just to the right of the first in Fig. 4, bounded by $g_{3 / 2}^{\mathrm{H}_{2} \mathrm{O}}<g^{\mathrm{H}_{2} \mathrm{O}} \leq g_{5 / 2}^{\mathrm{H}_{2} \mathrm{O}}$ and $0<g^{\mathrm{CO}_{2}} \leq g_{3 / 2} \mathrm{CO}_{2}$. Next the carbon dioxide rank is increased, resulting in a third region above the other two. This is continued until the entire two-dimensional space is allocated. At each step, the gas chosen for advancement is the one that results in the lowest-altitude peak cooling rate in the next region. In this way the regions are in order of the height at which they have their peak cooling.

A possible concern about the way $g$ space is allocated here is that it is based on the abundance of two gases in one particular atmosphere and, when applied to a profile in which the relative abundances are changed, the allocation would not necessarily still be appropriate. There are several points to make here. First, the $g$ intervals for each individual gas typically represent a difference of a factor 5 in $k$. Hence, the relative abundance of gases has to be very substantially changed to result in a significant difference in the optimum order in which regions are allocated. Second, in the case of water vapor and carbon dioxide, a change in the order in which the ranks of the two gases are incremented would mainly change the regions to which parts of the spectrum are allocated in the upper right of Fig. 4, where very little of the spectrum actually lies. Note that the first region in the lower left of this figure always has the same configuration, being bounded by $0<g^{\mathrm{H}_{2} \mathrm{O}} \leq g_{3 / 2}^{\mathrm{H}_{2} \mathrm{O}}$ and $0<g^{\mathrm{CO}_{2}} \leq g_{3 / 2} \mathrm{CO}_{2}$. Third, it would be a simple matter to split one or two of these regions into two or more subregions if they turned out to be particularly critical to the accuracy of the result when relative abundance was changed over a realistic range. In practice for present-day terrestrial atmospheres and under doubled $\mathrm{CO}_{2}$, this turns out not to be necessary.

This procedure outlined above is straightforward to implement for more than two gases; if we have $m$ gases, then at each step we have a choice of $m$ different ways in which the advancement can take place. This may be done by testing the altitude of the peak cooling rate in the new region in the case of advancement of each gas separately and choosing the one with the lowest altitude, or by some other method. Figure 5 illustrates the allocation of three-dimensional $g$ space for the overlapping of water vapor, carbon dioxide, and ozone. In the case of ozone, the number of $g$ intervals necessary to achieve a tolerance of $\sigma_{\max }=0.03 \mathrm{~K} \mathrm{day}^{-1}$ was $n_{g}{ }^{\mathrm{O}_{3}}=6$ (see Table 1). Thus, it can be seen that for the total number of regions required for $m$ gases is

$$
n_{g}=1-m+\sum_{i=1}^{m} n_{g}^{i},
$$




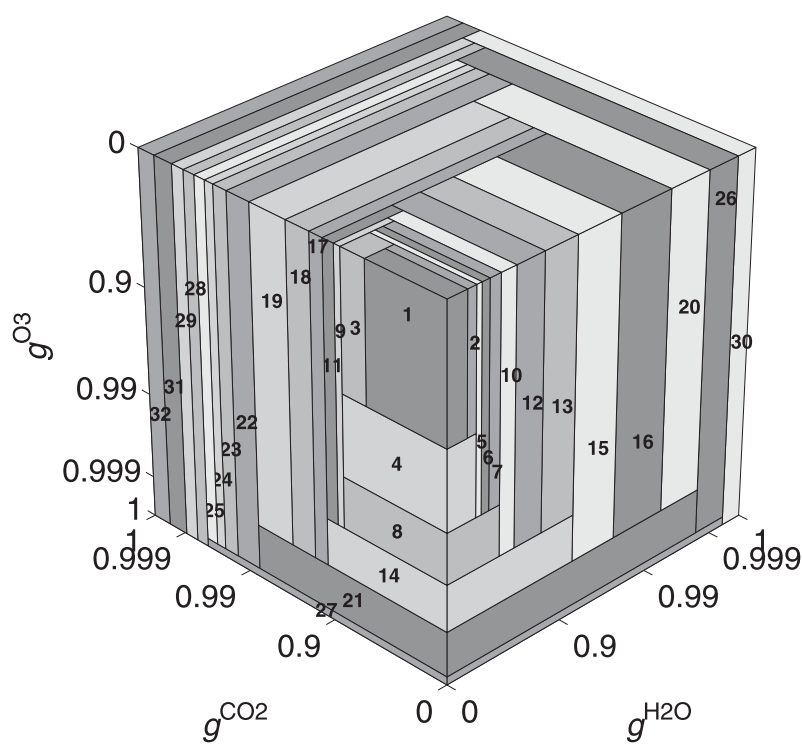

FIG. 5. Illustration of how three-dimensional $g$ space (for the gases $\mathrm{H}_{2} \mathrm{O}, \mathrm{CO}_{2}$, and $\mathrm{O}_{3}$ ) is divided up in the case of the 32-point model discussed in the text. Note that the upper surface shows the same divisions between $\mathrm{H}_{2} \mathrm{O}$ and $\mathrm{CO}_{2}$ as were shown in Fig. 4, except that here the axes have been scaled to reveal the regions confined to $g$ values very near 1 .

where $n_{g}^{i}$ is the number of $g$ intervals required for the $i$ th gas in isolation. For the remainder of this paper we use the term " $g$ interval" to indicate an interval for one particular gas (as shown in Table 1) but " $g$ region" for a cuboid in three-dimensional $g$ space (as shown in Fig. 5).

After all regions have been allocated, the most representative $k$ value must be selected for each active gas within each region, as a function of pressure. As in section 3, a first guess of the appropriate $k$ value for each region, gas, and reference pressure is calculated as the Planck-function-weighted mean of the high-resolution values of $\ln k$ within that region. The Planck function used is from the MLS standard atmosphere. We then use the framework of optimal estimation theory and define a "state vector" $\mathbf{x}$ containing the natural logarithm of $m \times n_{g}$ scaling factors, that is, one for each gas and region, which will scale the $k$ values at all pressures. The objective is to find the vector $\mathbf{x}$ that minimizes the difference in broadband heating rates and fluxes between the FSCK calculations and benchmark line-by-line calculations for $n_{p}$ "training" profiles, in a least squares sense. This is achieved by minimizing the following cost function:

$$
J=h \mathbf{x}^{\mathrm{T}} \mathbf{x}+\sum_{i=1}^{n_{p}} \sigma_{i}^{2},
$$

where $\sigma_{i}^{2}$ is the error variance of training profile $i$, as defined in (6) but for broadband variables, and quantifies the error in both the broadband heating rate profile and the broadband fluxes at the surface and the top of the atmosphere. The first term in (8) is present to ensure a stable and unique solution. It represents the squared deviation of the logarithm of the scaling factors away from zero and penalizes large deviations in the scaled $k$ values from the first guesses derived above. A small value for the weight $h$, somewhere between $10^{-6}$ and $10^{-5}$, leads to the best fit to the training profiles. The cost function is minimized using the Levenberg-Marquardt algorithm, a modification of the Gauss-Newton algorithm; both are described in detail by Rodgers (2000). This procedure requires calculation of the Jacobian matrix, which represents the partial derivative of all the heating rates and fluxes required to calculate $\sigma^{2}$ with respect to all the elements of $\mathbf{x}$. This is done numericallythat is, by perturbing in turn each of the $m \times n_{g}$ values of $\mathbf{x}$ by a small amount, calculating the perturbed $k$ profile for that particular region, and running a pseudomonochromatic radiative transfer calculation for each of the $n_{p}$ profiles. For $n_{g}=32, m=3$, and $n_{p}=4$, this whole minimization process can be computed in much less than a minute on a typical workstation, even when many iterations are required.

The extension to other gases such as methane and nitrous oxide should be straightforward using a similar method: by adding further dimensions to the cube shown in Fig. 5 and partitioning the resulting "hypercube" in the same way. Further work will be needed to confirm that the sensitivity to trace-gas concentrations can be calculated accurately. Note that for weather forecasting it is generally not necessary to represent changes to trace gases during the simulation, so one may combine all wellmixed gases with carbon dioxide into a "composite" gas (e.g., Ritter and Geleyn 1992). Curry et al. (2006) showed that the global-mean temperature error resulting from assuming methane and nitrous oxide to be well mixed is less than $0.2 \mathrm{~K}$ below $30 \mathrm{~km}$, rising to $1 \mathrm{~K}$ above $50 \mathrm{~km}$, while the error due to treating CFC11 and CFC12 as well mixed is less than $0.1 \mathrm{~K}$ everywhere below $50 \mathrm{~km}$. These small errors will have no detectable impact on the accuracy of weather forecasts. For the comparisons in the remainder of this paper we therefore use primarily the three-gas model described in this section, which utilizes the $g$ intervals listed in Table 1 and requires a total of 32 quadrature points. Comparisons are also performed with a faster but less accurate model consisting of 23 quadrature points, obtained by using a heating rate tolerance for the individual gases of $\sigma_{\max }=0.05 \mathrm{~K} \mathrm{day}^{-1}$, which results in $n_{g}^{\mathrm{H}_{2} \mathrm{O}}=10, n_{g}^{\mathrm{CO}_{2}}=10$ and $n_{g}^{\mathrm{O}_{3}}=5$.

The range of applicability of an FSCK radiation scheme (and indeed most CKD schemes) is entirely dependent on the range of temperature and gas concentrations in the 

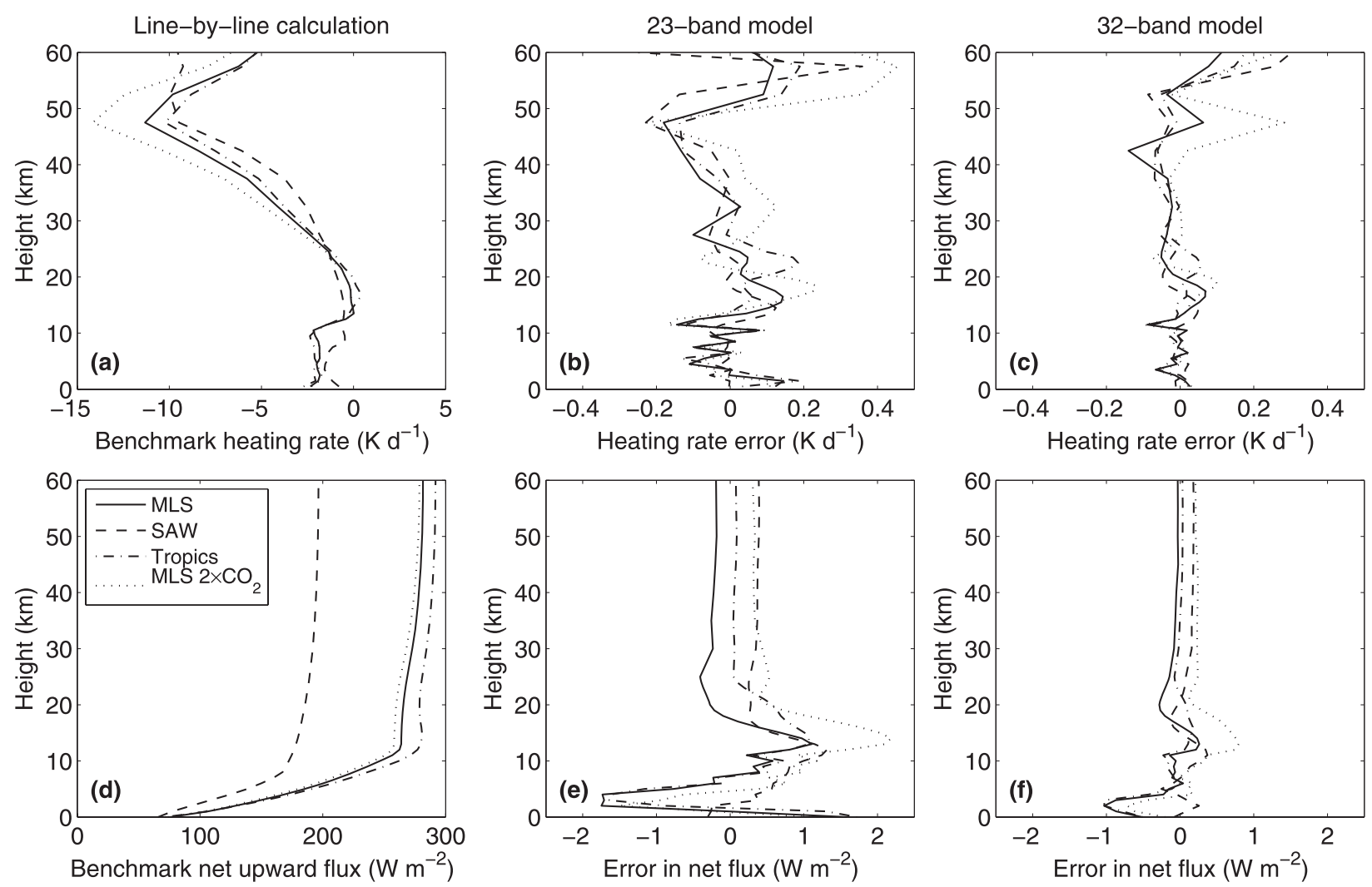

FIG. 6. Evaluation of the 23- and 32-point FSCK models described in the text on four different McClatchey et al. (1972) standard atmospheres containing only $\mathrm{H}_{2} \mathrm{O}, \mathrm{CO}_{2}$, and $\mathrm{O}_{3}$. These profiles were used as the training dataset to derive the two FSCK models. (a) Longwave heating rate calculated at full spectral resolution, (b) the error in heating rate of the 23-point FSCK model, (c) the error in heating rate of the 32-point FSCK model. (d)-(f) As in (a)-(c) but for net longwave flux (upwelling minus downwelling). [Standard atmospheres are indicated by the legend in (d) where MLS is the midlatitude summer atmosphere and SAW is the subarctic winter atmosphere.]

training profiles. Therefore, for paleoclimate simulations or the atmospheres of other planets, a different set of training profiles would need to be used. In general, the wider the intended applicability of the scheme, the more regions into which it is likely to be necessary to partition $g$ space to achieve the same accuracy. Conversely, for short-term terrestrial weather forecasts where carbon dioxide and trace gas concentrations may be held fixed, there is no need to bear the extra computational cost of using a climate-quality scheme that allocates many $g$ points to ensure the correct climate sensitivity to changes in each gas.

\section{Evaluation using different atmospheres}

In this section the 23-point and 32-point FSCK models developed in the previous section are evaluated by comparing them to line-by-line calculations. The optimum settings for these models are derived using four training profiles and then tested on four other profiles. Clearly for an operational radiation scheme one would need to use a wider range of training profiles. For simplicity, we use the same mass absorption spectra for the set of reference pressures discussed in section 3 , with the assumption that in an operational version of the model the additional dependence of $k$ on temperature and water vapor mixing ratio could be represented accurately either parametrically (e.g., Fu and Liou 1992) or as a multidimensional lookup table (e.g., Mlawer et al. 1997).

Figures $6 \mathrm{a}$ and $6 \mathrm{~d}$ show the benchmark heating rate and net upward flux profile for the four training profiles, as calculated using a line-by-line model with a spectral resolution of $0.0025 \mathrm{~cm}^{-1}$. Corresponding values of outgoing longwave radiation are given in Table 2. The benchmark heating rate and net flux profiles are very similar to those shown in other studies, although comparing to the line-by-line model of Mlawer et al. (1997) it can be seen that our TOA net flux is less by around $1.5 \mathrm{~W} \mathrm{~m}^{-2}$. This is likely to be related to the absence of other absorbing gases (particularly methane) in our simulations, and the fact that Mlawer et al. (1997) performed calculations in the range $10-3000 \mathrm{~cm}^{-1}$ whereas 
TABLE 2. Benchmark calculations of the outgoing longwave radiation and the associated error in the equivalent calculation of the 23- and 32-point FSCK models $\left(\mathrm{W} \mathrm{m}^{-2}\right)$ for a $\mathrm{CO}_{2}$ concentration of 350 ppmv. Five McClatchey et al. (1972) standard atmospheres have been used: midlatitude summer (MLS), subarctic winter (SAW), tropics, midlatitude winter (MLW), and subarctic summer (SAS). The last two were not part of the training dataset: the results of the FSCK models for these are shown in bold.

\begin{tabular}{lccc}
\hline \hline Profile & Benchmark & 23-pt error & 32-pt error \\
\hline MLS & 281.75 & -0.18 & -0.03 \\
SAW & 196.69 & +0.41 & +0.19 \\
Tropics & 291.89 & +0.09 & +0.04 \\
MLW & 228.21 & $+\mathbf{0 . 1 7}$ & $+\mathbf{0 . 0 8}$ \\
SAS & 262.43 & $-\mathbf{0 . 4 8}$ & $\mathbf{- 0 . 1 6}$ \\
\hline
\end{tabular}

we limited our calculations to the range $100-2500 \mathrm{~cm}^{-1}$. They also used a different absorption database.

Figures $6 \mathrm{~b}$ and $6 \mathrm{e}$ depict the heating-rate and net-flux errors for the 23-point FSCK model. The root-meansquare heating-rate error of this model over all four profiles is $0.1 \mathrm{~K} \mathrm{day}^{-1}$ when weighted by the square root of pressure as in (6). Figure $6 \mathrm{~b}$ shows that this is made up of a smaller error in the troposphere and lower stratosphere but an error up to $0.4 \mathrm{~K} \mathrm{day}^{-1}$ at the stratopause for two of the profiles. Of course, the magnitude of the heating rate is substantially larger here; in percentage terms this error is only $4 \%$. The corresponding errors in outgoing longwave radiation are listed in Table 2 . These errors are considerably less than the 23-point CKD model of Fomin (2004).

Figures $6 \mathrm{c}$ and $6 \mathrm{f}$ show the errors for the 32-point model. This time the root-mean-square heating-rate error is only $0.04 \mathrm{~K} \mathrm{day}^{-1}$, and Fig. $6 \mathrm{f}$ shows that the net flux is accurate to better than $1 \mathrm{~W} \mathrm{~m}^{-1}$ at all heights. The errors are generally a little smaller than those of the 256-point RRTM model (Mlawer et al. 1997).

A fairer test of the two FSCK models is given in Fig. 7, which uses four profiles not among the set of four training profiles. This time the heating rate errors below $50 \mathrm{~km}$ are largely unchanged, although the errors at $60 \mathrm{~km}$ are larger for both models. Errors in the flux profiles are also increased, but still less than around $1 \mathrm{~W} \mathrm{~m}^{-2}$ at all heights for the 32-point model and with errors in outgoing longwave radiation less than $0.5 \mathrm{~W} \mathrm{~m}^{-2}$. The errors in outgoing longwave radiation for the two new profiles with twentieth-century $\mathrm{CO}_{2}$ levels are given in bold in Table 2. The ability of the 32-point model to reliably simulate profiles with very different gas abundances indicates that the order in which the three different gases were selected in the partitioning of $g$ space in section 4 is not critical for the performance.

Finally we consider an important scenario for climate change forecasts in which the mixing ratio of carbon dioxide is doubled to $700 \mathrm{ppmv}$. Figures $7 \mathrm{~b}$ and $7 \mathrm{c}$ reveal
TABLE 3. Benchmark calculations of the change to the outgoing longwave radiation associated with doubling $\mathrm{CO}_{2}$ concentrations for three of the atmospheres used in Table 2, together with the percentage error in the 23- and 32-point FSCK calculations of this change. In the case of the subarctic winter (SAW) and tropical profiles, the doubled $\mathrm{CO}_{2}$ benchmarks were not used as training profiles: these percentages are shown in bold to indicate that they are a less constrained test of the method.

\begin{tabular}{lccc}
\hline \hline Profile & Benchmark & 23-pt error & 32-pt error \\
\hline MLS & $2.87 \mathrm{~W} \mathrm{~m}^{-2}$ & $-17 \%$ & $-8 \%$ \\
SAW & $1.82 \mathrm{~W} \mathrm{~m}^{-2}$ & $\mathbf{- 2 9 \%}$ & $-\mathbf{1 2} \%$ \\
Tropics & $3.31 \mathrm{~W} \mathrm{~m}^{-2}$ & $\mathbf{- 2 0} \%$ & $-\mathbf{1 0} \%$ \\
\hline
\end{tabular}

that the profiles with doubled $\mathrm{CO}_{2}$ tend to have larger heating rate errors at the stratopause. Table 3 shows the reduction in outgoing longwave radiation due to doubled $\mathrm{CO}_{2}$ calculated using the highest spectral resolution for three of the profiles, together with the percentage error in the two FSCK models. In the case of the midlatitude summer atmosphere, both the standard and double $\mathrm{CO}_{2}$ profiles were part of the training set, and the 32-point model is able to capture the change with only an $8 \%$ error. This rises by a few percent for the subarctic winter and tropical profiles, which represent a more objective test of the model since only the standard $\mathrm{CO}_{2}$ concentrations were used in the training datasets. The errors are considerably larger for the 23-point model, suggesting that, without further refinement at least, the lower-resolution model would not be reliable for use in a climate model although it would be satisfactory for weather forecasts.

\section{Conclusions}

This paper has investigated the possibility of substantially increasing the speed of the longwave part of radiation schemes used in GCMs by abandoning the practice of splitting the spectrum up into bands and, rather, performing the correlated- $k$ method across the full spectrum. In section 1 , three reasons were cited as to why the longwave spectrum ought to be divided up into bands. The first of these-that the Planck function should vary little across a band-was overcome in section $2 \mathrm{c}$ by the use of an effective Planck function, and demonstrated in practice in section 3 . In an operational radiation code, the effective Planck function would be precomputed versus temperature for each of the $n_{g}$ quadrature points, and then implemented efficiently as a temperature-dependent lookup table.

The second motivation for bands concerned the need to minimize the number of active gases per band and (for some schemes) to assume random overlap of the different spectra. In sections 4 and 5 it was shown that 

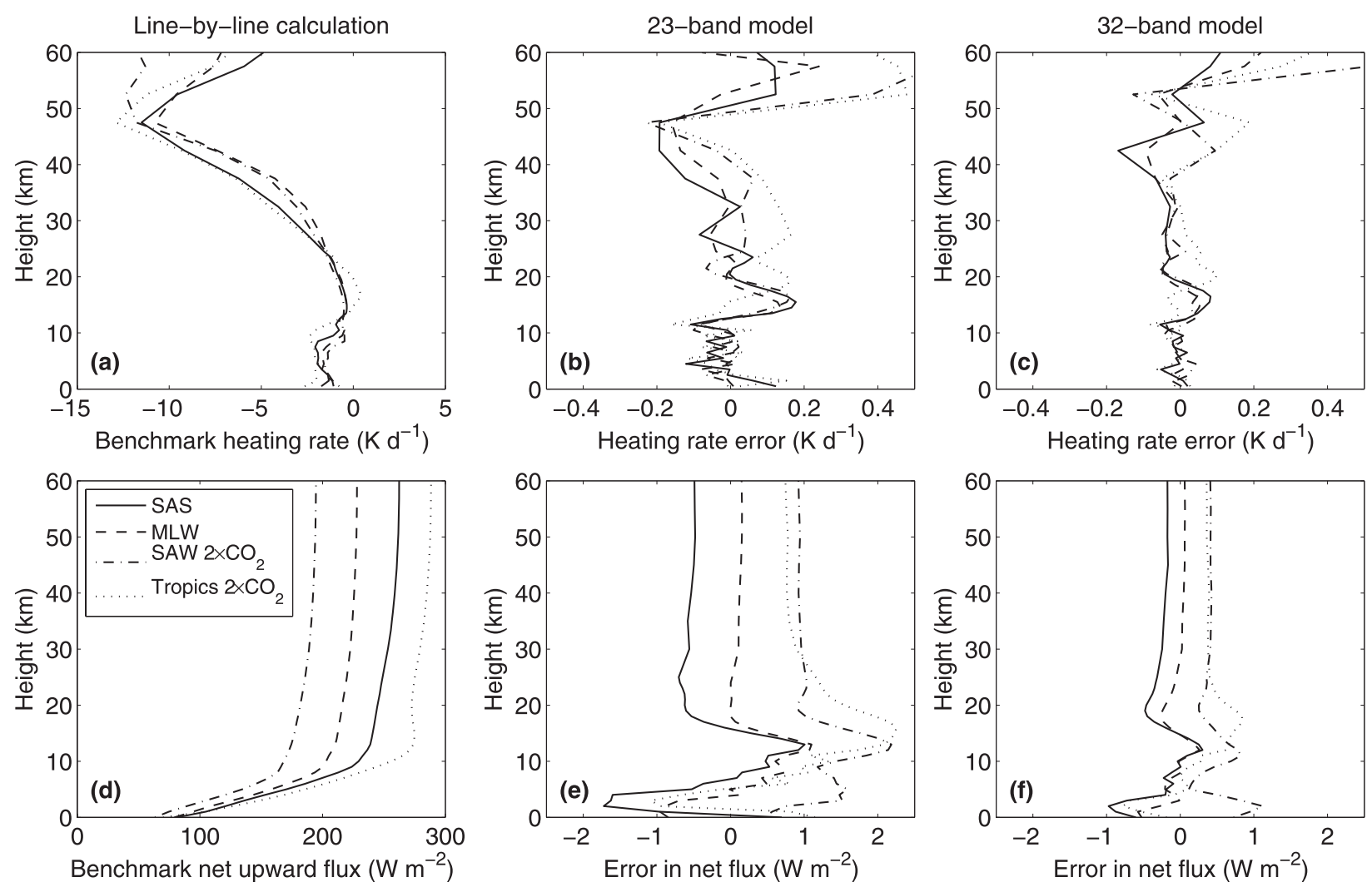

FIG. 7. As in Fig. 6 but for four profiles not used to train the two models: the McClatchey et al. (1972) subarctic summer (SAS) and midlatitude winter (MLW) atmospheres and two of the profiles from Fig. 6 but with doubled $\mathrm{CO}_{2}$ concentrations.

this obstacle could be overcome without a large computational cost by partitioning multidimensional $g$ space as illustrated in Fig. 5. It has been shown that a total of $\sim 32$ quadrature points is sufficiently accurate for most applications (in clear-sky conditions with the three dominant absorbing gases).

The third consideration was the need to represent the spectrally varying properties of clouds and aerosols. This issue has not been tackled in this paper, yet is a potential concern since each pseudomonochromatic radiation calculation can represent widely spaced parts of the spectrum and therefore be unable to resolve the slow but significant spectral variation of the optical properties of atmospheric particles. For low clouds, the impact of the clouds on fluxes and heating rates is concentrated almost exclusively in the atmospheric infrared window (8-13 $\mu \mathrm{m})$, so only the modest spectral variation across this range will play a role. Moreover, preliminary calculations with layers of optically thick cloud indicate that fewer than half of the 32 individual $g$ regions are sensitive to clouds and, in almost all of those, the heating rate is dominated by the contribution from less than $250 \mathrm{~cm}^{-1}$ of the spectrum. Therefore, for each region it may be possible to calculate the effective particulate scattering properties by averaging them over the parts of the spectrum that contribute to that region but weighting each by the expected contribution to the heating rate in a selection of representative cloudy profiles. Clearly, further work is required to verify this. For improved efficiency in a GCM, these ideas could be coupled to the method of Manners et al. (2009), in which those bands that are insensitive to clouds are computed much less frequently than the fewer number of cloud-sensitive bands.

It should be stressed that the analysis performed in this paper is very much in the form of a "proof of concept": to build an operational FSCK longwave radiation scheme, the following further work would be necessary:

- Use high-resolution absorption spectra over a wide range of pressure, temperature, and water vapor mixing ratio to parameterize correctly the dependence of the representative $k$ value in each $g$ region on these parameters, including the contribution of the water vapor continuum.

- Add the contributions of the other active trace gases, particularly methane, nitrous oxide, CFC11, and CFC12. For weather forecasting these gases can be considered vertically well mixed, so it is valid to combine them with 
carbon dioxide into a single composite gas (e.g., Ritter and Geleyn 1992). However, for climate applications and decade-long reanalysis integrations they need to be represented explicitly.

- Implement FSCK in a fully scattering radiation code with lookup tables for the effective Planck function and the appropriately averaged cloud and aerosol optical properties in each $g$ region. It may be necessary to split a few of the regions into two if particulate scattering properties vary too much within a region.

Application of FSCK with an effective Planck function is not limited to one-dimensional radiation schemes used in GCMs. The formulation presented in section 2 applies equally well in three dimensions, with the $j$ index in the various equations now indicating the summation over all points in 3D space. The method is also applicable to radiative transfer in other fields.

Acknowledgments. The high-resolution absorption spectra were calculated using the HITRAN database by Igor Ptashnik. I thank him, Keith Shine, and Graeme Stephens for valuable discussions in the course of this work.

\section{APPENDIX}

\section{Formulation of the Weighting Matrix $\mathbf{W}_{j i}$}

In this appendix it is shown how we may calculate the elements of the weighting matrix $\mathbf{W}_{j i}$ used in (1). Note that the use of a weighting matrix in section 2 is simply to facilitate the demonstration that the full-spectrum correlated- $k$ (FSCK) method is mathematically valid; it does not mean that subsequent application of FSCK need include explicit calculation of $\mathbf{W}_{j i}$. Since section 2 deals with the spectral integration, here we consider only a narrow part of the spectrum, but for brevity the dependence of all the terms on wavenumber $\eta$ is not written.

The spectral heating rate $\left[\mathrm{K} \mathrm{s}^{-1}\left(\mathrm{~cm}^{-1}\right)^{-1}\right]$ at height $z$ may be written in terms of the vertical divergence of the net upward spectral flux $F=F^{\uparrow}-F^{\downarrow}\left[\mathrm{W} \mathrm{m}^{-2}\left(\mathrm{~cm}^{-1}\right)^{-1}\right]$ as

$$
\dot{T}(z)=-\frac{1}{\rho(z) C_{p}} \frac{d F}{d z}
$$

where $\rho(z)$ is the density of the air at height $z$ and $C_{p}$ is the specific heat capacity at constant pressure. If we discretize the atmosphere into $n_{z}$ layers, each thin enough that the Planck function can be considered to be vertically constant within them, then the heating rate of layer $i$ with thickness $\Delta z_{i}$ may be rewritten as

$$
\dot{T}_{i}=\frac{F_{i-1 / 2}^{\uparrow}-F_{i+1 / 2}^{\uparrow}+F_{i+1 / 2}^{\downarrow}-F_{i-1 / 2}^{\downarrow}}{\rho_{i} C_{p} \Delta z_{i}} .
$$

The upwelling flux leaving the top of the layer is the sum of the flux emitted from the layer and the transmitted fraction of the upwelling flux entering the base of the layer, and similarly for the downwelling flux leaving the base of the layer:

$$
\begin{aligned}
& F_{i+1 / 2}^{\uparrow}=B_{i} \varepsilon_{i}+\tau_{i} F_{i-1 / 2}^{\uparrow}, \\
& F_{i-1 / 2}^{\downarrow}=B_{i} \varepsilon_{i}+\tau_{i} F_{i+1 / 2}^{\downarrow},
\end{aligned}
$$

where $B_{i}$ is the Planck function of layer $i\left[\mathrm{~W} \mathrm{~m}{ }^{-2}\left(\mathrm{~cm}^{-1}\right)^{-1}\right]$, $\varepsilon_{i}$ is the emissivity of the layer, and $\tau_{i}=1-\varepsilon_{i}$ is its transmissivity. It was shown by Elsasser (1942) and verified by Rodgers and Walshaw (1966), among others, that in the longwave part of the spectrum the emissivity may be approximated as $\varepsilon_{i}=1-\exp \left(-D \delta_{i}\right)$, where $D=1.66$ is known as the diffusivity factor and $\delta_{i}$ is the absorption optical depth of the layer. Substitution of (A3) into (A2) yields

$$
\rho_{i} C_{p} \Delta z_{i} \dot{T}_{i}=\varepsilon_{i}\left(F_{i-1 / 2}^{\uparrow}+F_{i+1 / 2}^{\downarrow}-2 B_{i}\right) .
$$

In a nonscattering atmosphere, the downwelling flux at the layer top may be expressed as the sum of the emission from all layers above:

$$
F_{i+1 / 2}^{\downarrow}=\sum_{j=i+1}^{n_{z}} \varepsilon_{j} B_{j} \tau_{i j}
$$

where $\tau_{i j}$ is the combined transmissivity of atmospheric layers between $i$ and $j$ (but not including layers $i$ and $j$ themselves), equal to the product of the individual layer transmissivities; that is,

$$
\tau_{i j}=\prod_{k=i+1}^{j-1} \tau_{k}
$$

Likewise, the upwelling flux at the base of the layer may be expressed in terms of the emission from all layers below it and the emission from the surface:

$$
F_{i-1 / 2}^{\uparrow}=\varepsilon_{0} B_{0} \tau_{0 i}+\sum_{j=1}^{i-1} \varepsilon_{j} B_{j} \tau_{j i},
$$

where $\varepsilon_{0} B_{0}$ is the flux emitted from the surface, and for the moment scattering from the surface is neglected. We are now in a position to derive $\mathbf{W}_{j i}$ in (1). It is defined for source layers $j \in\left\{0,1, \ldots, n_{z}\right\}$ (including the surface at 
$j=0)$ and target layers $i \in\left\{1,2, \ldots, n_{z}\right\}$. Equating (1) with (A4)-(A6) requires that

$$
\rho_{i} C_{p} \Delta z_{i} \mathbf{W}_{j i}= \begin{cases}\varepsilon_{j} \varepsilon_{i} \tau_{j i}, & j<i \\ -2 \varepsilon_{i}, & j=i \\ \varepsilon_{j} \varepsilon_{i} \tau_{i j}, & j>i .\end{cases}
$$

This formulation for longwave radiative transfer may be extended to scattering atmospheres, although it is a little more cumbersome. Defining the albedo of layer $i$ as $\alpha_{i}$ and redefining the transmissivity as $\tau_{i}=1-\varepsilon_{i}-\alpha_{i}$, the effect of allowing a single scattering event between emission at $j$ and absorption at layer $i$ may be calculated by summing over all possible scattering layers $k$ (with $k=0$ corresponding to scattering from the surface). In the case of $j<i$ we may have scattering from layers above layer $i$ and below layer $j$ (forward scattering by a layer lying between $i$ and $j$ would be included in the definition of the transmissivity). Thus, the first condition of (A7) is redefined as

$$
\rho_{i} C_{p} \Delta z_{i} \mathbf{W}_{j<i}=\varepsilon_{j} \varepsilon_{i}\left(\tau_{j i}+\sum_{k=0}^{j-1} \alpha_{k} \tau_{k j} \tau_{k i}+\sum_{k=i+1}^{n_{z}} \alpha_{k} \tau_{j k} \tau_{i k}\right)
$$

and similarly for the other two conditions of (A7). The two transmissivities in each summation represent the transmissivity between the emission event and the scattering event, and between the scattering event and the absorption event. Higher-order scattering can be represented by multiple summations.

\section{REFERENCES}

Ambartzumian, V., 1936: The effect of the absorption lines on the radiative equilibrium of the outer layers of the stars. $P u b l$. Obs. Astron. Univ. Leningrad, 6, 7-18.

Cahalan, R. F., W. Ridgeway, W. J. Wiscombe, T. L. Bell, and J. B. Snider, 1994: The albedo of fractal stratocumulus clouds. J. Atmos. Sci., 51, 2434-2455.

Clough, S. A., M. W. Shephard, E. J. Mlawer, J. S. Delamere, M. J. Iacono, K. Cady-Pereira, S. Boukabara, and P. D. Brown, 2005: Atmospheric radiative transfer modeling: A summary of the AER codes. J. Quant. Spectrosc. Radiat. Transfer, 91, 233-244.

Curry, C. L., N. A. McFarlane, and J. F. Scinocca, 2006: Relaxing the well-mixed greenhouse gas approximation in climate simulations: Consequences for stratospheric climate. J. Geophys. Res., 111, D08104, doi:10.1029/2005JD006670.

Edwards, J. M., 1996: Efficient calculation of infrared fluxes and cooling rates using the two-stream equations. J. Atmos. Sci., 53, 1921-1932.

Elsasser, W. M., 1942: Heat Transfer by Infrared Radiation in the Atmosphere. Harvard Meteorological Studies, Vol. 6, Harvard University Press, 106 pp.
Eymet, V., J. L. Dufresne, P. Ricchiazzi, R. Fournier, and S. Blanco, 2004: Long-wave radiative analysis of cloudy scattering atmospheres using a net exchange formulation. Atmos. Res., 72, 239-261.

Fomin, B. A., 2004: A $k$-distribution technique for radiative transfer simulation in inhomogeneous atmosphere: 1. FKDM, fast $k$-distribution model for the longwave. J. Geophys. Res., 109, D02110, doi:10.1029/2003JD003802.

Fu, Q., and K. N. Liou, 1992: On the correlated $k$-distribution method for radiative transfer in nonhomogenous atmospheres. J. Atmos. Sci., 49, 2139-2156.

,-- M. C. Cribb, T. P. Charlock, and A. Grossman, 1997: Multiple scattering parameterization in thermal infrared radiative transfer. J. Atmos. Sci., 54, 2799-2812.

Green, J. S. A., 1967: Division of radiative streams into internal transfer and cooling to space. Quart. J. Roy. Meteor. Soc., 93, 371-372.

Hansen, J., G. Russell, D. Rind, P. Stone, A. Lacis, S. Lebedeff, R. Ruedy, and L. Travis, 1983: Efficient three-dimensional global models for climate studies: Models I and II. Mon. Wea. Rev., 111, 609-662.

Lacis, A., and V. Oinas, 1991: A description of the correlated $k$-distribution method for modeling nongray gaseous absorption, thermal emission, and multiple scattering in vertically inhomogeneous atmospheres. J. Geophys. Res., 96, 90279063.

_ W. C. Wang, and J. Hansen, 1979: Correlated $k$-distribution method for radiative transfer in climate models: Application to effect of cirrus clouds on climate. NASA Conf. Publication 2076, 309-314.

Manners, J., J.-C. Thelen, J. Petch, P. Hill, and J. M. Edwards, 2009: Two fast radiative transfer methods to improve the temporal sampling of clouds in numerical weather prediction and climate models. Quart. J. Roy. Meteor. Soc., 135, 457-468.

McClatchey, R. A., R. W. Fenn, J. E. A. Selby, F. E. Volz, and J. S. Garing, 1972: Optical properties of the atmosphere. 3rd ed. Air Force Cambridge Research Laboratories Rep. AFCRL720497, 108 pp.

Mitsel, A. A., I. V. Ptashnik, K. M. Firsov, and A. B. Fomin, 1995: Efficient technique for line-by-line calculating the transmittance of the absorbing atmosphere. Atmos. Oceanic Opt., 8, 847-850.

Mlawer, E. J., S. J. Taubman, P. D. Brown, M. J. Iacono, and S. A. Clough, 1997: Radiative transfer for inhomogeneous atmospheres: RRTM, a validated correlated- $k$ model for the longwave. J. Geophys. Res., 102, 16 663-16 682.

Modest, M. F., and H. Zhang, 2002: The full-spectrum correlated- $k$ distribution for thermal radiation from molecular gas-particulate mixtures. J. Heat Transfer, 124, 30-38.

Morcrette, J.-J., 2000: On the effects of the temporal and spatial sampling of radiation fields on the ECMWF forecasts and analyses. Mon. Wea. Rev., 128, 876-887.

—, H. W. Barker, J. N. S. Cole, M. J. Iacono, and R. Pincus, 2008: Impact of a new radiation package, McRad, in the ECMWF Integrated Forecasting System. Mon. Wea. Rev., 136, 47734798.

Oinas, V., A. A. Lacis, D. Rind, D. T. Shindell, and J. E. Hansen, 2001: Radiative cooling by stratospheric water vapor: Big differences in GCM results. Geophys. Res. Lett., 28, 27912794.

Pawlak, D. T., E. E. Clothiaux, M. F. Modest, and J. N. S. Cole, 2004: Full-spectrum correlated- $k$ distribution for shortwave atmospheric radiative transfer. J. Atmos. Sci., 61, 2588-2601. 
Ritter, B., and J.-F. Geleyn, 1992: A comprehensive radiation scheme for numerical weather prediction models with potential applications in climate simulations. Mon. Wea. Rev., 120, 303-325.

Rodgers, C. D., 2000: Inverse Methods for Atmospheric Sounding: Theory and Practice. World Scientific, $238 \mathrm{pp}$.

, and C. D. Walshaw, 1966: The computation of infrared cooling rate in planetary atmospheres. Quart. J. Roy. Meteor. Soc., 92, 67-92.
Rothman, L. S., and Coauthors, 2004: The HITRAN 2004 molecular spectroscopic database. J. Quant. Spectrosc. Radiat. Transfer, 96, 139-204.

Shonk, J. K. P., and R. J. Hogan, 2008: Tripleclouds: An efficient method for representing cloud inhomogeneity in $1 \mathrm{D}$ radiation schemes by using three regions at each height. J. Climate, 21, 2352-2370.

Yang, G.-Y., and J. M. Slingo, 2001: The diurnal cycle in the tropics. Mon. Wea. Rev., 129, 784-801. 